\title{
Three-dimensional structure of neuropeptide $Y$ pre-pro-peptide to reveal its interaction with lipid membrane
}

\author{
Li Xing, Ming-Siao Hsiao, Zhi-Feng Kuang, Yen Ngo, Steve Kim, and Lawrence F. Drummy \\ Materials and Manufacturing Directorate, Air Force Research Laboratory, WPAFB OH 45433
}

Neuropeptide Y (NPY) is one of the abundant proteins within brain [1], and it is involved in regulation of important biological and pathophysiological functions such as food uptake, energy homeostasis, circadian rhythm and cognition. NPY also serves as a major neurochemical component in stress response and a key element in modulation of emotional-affective behavior. NPY is therefore a potential drug for the disease condition characterized by dysregulation of NPY-dependent physiological pathway(s), eg, resilience from traumatic conditions, or an indicator of susceptibility to stressful or threat-related events.

Mature NPY is a 36-amino acid peptide that belongs to the family of gut-brain peptides [2]. Mature NPY contains an amphipathic alpha-helix that promotes binding to lipid membranes with the side chain of aromatic residues inserted into the hydrophobic region [3]. NPY is translated from mRNA as pre-propeptide of 97 amino acids and inserts its signal peptide into endoplasmic reticulum where the signal peptide is removed. The pro-peptide is then packaged in the Golgi apparatus into large-dense core vesicles where the pro-peptides are proteolytically processed and amidated before releasing as mature NPY peptide. NPY pre-pro-peptide can be obtained by translation of NPY mRNA in wheat-germ cellfree lysate in presence of liposomes and immunoprecipitated with antiserum against NPY [4]. So far, there is no structural information on the tertiary folding of NPY pre-pro-peptide or the quaternary engagement of NPY pre-pro-peptide with the lipid membrane, one of the key elements in NPY maturation. In this project, we aim to provide the electron microscopic density map of recombinant NPY pre-pro-peptide tagged with glutathione S-transferase (GST-NPY full length protein) in frozen hydrated ice.

Frozen hydrated specimens of recombinant GST-NPY full length protein were prepared onto Quantifoil holey-carbon grids and examined in a Philip CM200 transmission electron microscope using a Gatan 914 cryo-transfer holder. The micrographs were recorded on a CCD camera at a magnification of 20000x, corresponding to approximately $6.2 \AA$ per pixel at specimen space. An image-corrected FEI Titan TEM operating at $300 \mathrm{kV}$ and equipped with a Gatan K2-Summit direct electron detection device was also used to acquire cryo-EM data with enhanced signal-to-noise ratio for reconstructing the threedimensional structure.

The cryo-images of individual particles composed of GST-NPY full length showed as either donutshaped projections of $20 \mathrm{~nm}$ in diameter or arch-shaped projections with a dimension of $10 \mathrm{~nm}$ x $20 \mathrm{~nm}$ (Fig 1). The particle projections display light contrast at the center region, however, the contrast of central region appears denser than the vitreous ice at background. Structure predication using the NMR structure of mature NPY (PDB code 1RON) as template indicates that NPY pre-pro-peptide adapts into multiple alpha-helixes. The N-terminal helix from residue Lys5 to Gly27 constitutes the putative signal peptide and connected to the C-terminal helix via the amphipathic alpha-helix of mature NPY and flexible loops. Although the protein adapted into globular folding, the hydrophobic surface of the $\mathrm{N}$ terminal signal peptide is still solvent-accessible (Fig 1) and energetically unstable in buffer solution. We therefore believe that lipid membrane is involved in constitution of GST-NPY full length protein 
and a determination of the cryo-EM structure of GST-NPY full length protein complex is currently underway, with the goal of revealing the quaternary arrangement of alpha-helices with the lipid membrane.

\section{References:}

1. T.J. Sajdyk, A. Shekhar, and D.R. Gehlert. Neuropeptides 38 (2004), p225.

2. P. Holzer, F. Reichmann, and A. Farzi. Neuropeptides 46 (2012), p261.

3. R. Bader, A. Bettio, A.G. Beck-Sickinger, and O. Zerbe. J. Mol. Biol. 305 (2001), p307.

4. C.D. Minth, S.R. Bloom, J.M. Polak, and J.E. Dixon. Proc. Natl. Acad. Sci. 81 (1984), p4577.

5. The authors acknowledge funding from the Air Force Research Laboratory.
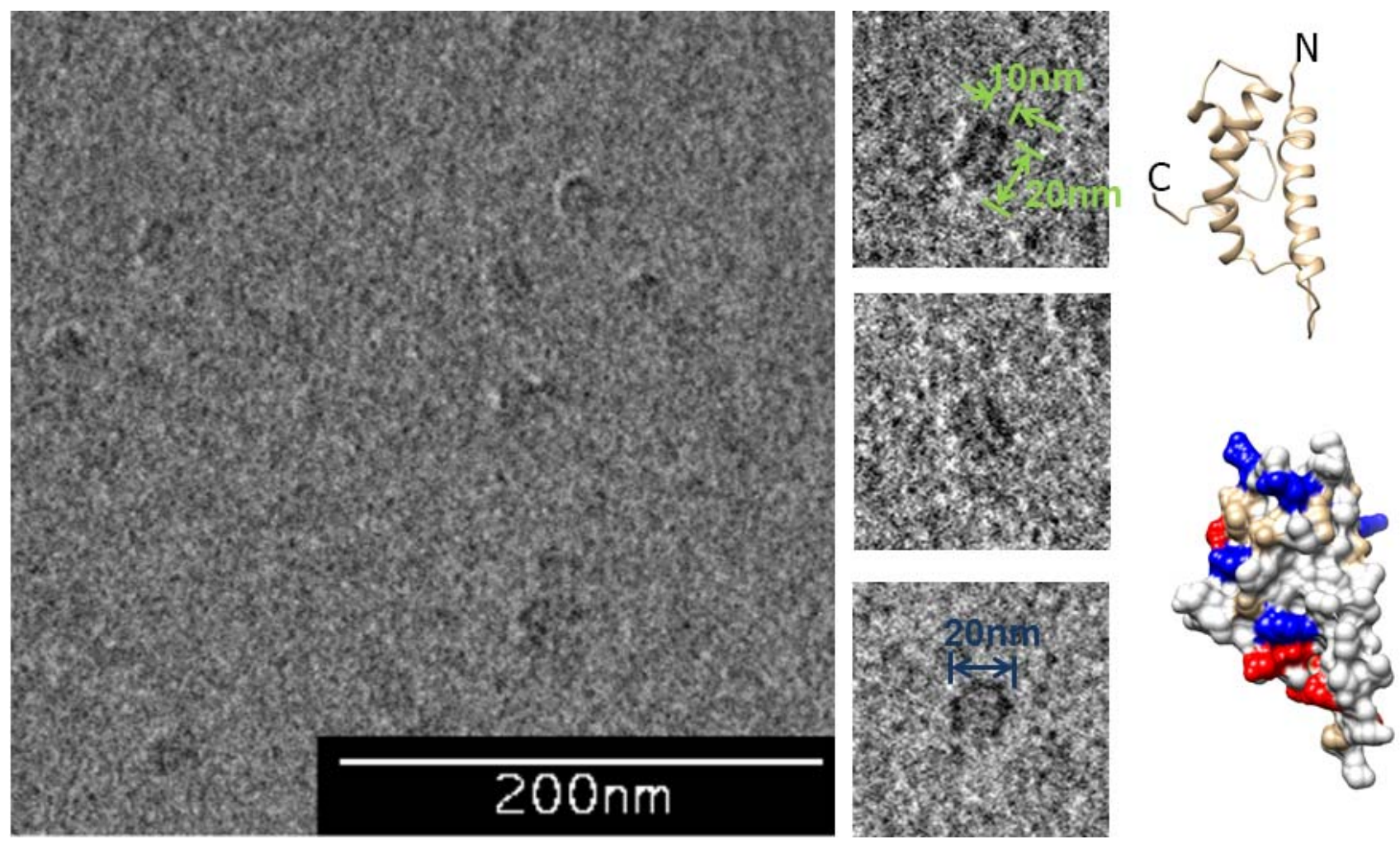

Figure 1. The cryo-electron micrograph (left panel) and the selected images (central column) reveal the morphology and size of GST-NPY full length proteins. The predicted structure of NPY pre-pro-peptide is displayed as ribbon drawing (top in right panel) to show the alpha-helixes and as surface rendering (bottom in right panel) to show the positively charged residues (blue), negatively charged residues (red), and hydrophobic residues (grey). The structure is displayed at the same orientation with the letter $\mathrm{N}$ and $\mathrm{C}$ labeling the corresponding terminus. 